\title{
Simulations of radio-wave anisotropic scattering to interpret type III radio burst data from Solar Orbiter, Parker Solar Probe, STEREO, and Wind
}

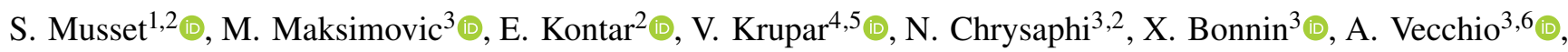

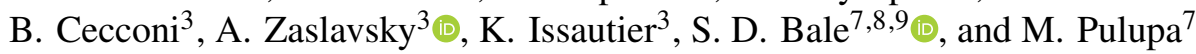 \\ 1 European Space Agency (ESA), European Space Research and Technology Centre (ESTEC), Keplerlaan 1, 2201 AZ Noordwijk, \\ The Netherlands \\ e-mail: sophie.musset@esa.int \\ 2 School of Physics \& Astronomy, University of Glasgow, Glasgow G12 8QQ, UK \\ ${ }^{3}$ LESIA, Observatoire de Paris, Université PSL, CNRS, Sorbonne Université, Université de Paris, 5 place Jules Janssen, \\ 92195 Meudon, France \\ ${ }^{4}$ Goddard Planetary Heliophysics Institute, University of Maryland, Baltimore County, Baltimore, MD, USA \\ 5 Heliophysics Science Division, NASA Goddard Space Flight Center, Greenbelt, MD, USA \\ 6 Radboud Radio Lab, Department of Astrophysics/IMAPP - Radboud University, PO Box 9010, 6500 GL Nijmegen, \\ The Netherlands \\ 7 Space Sciences Laboratory, University of California, Berkeley, CA, USA \\ 8 Physics Department, University of California, Berkeley, CA, USA \\ 9 Stellar Scientific (now HELIOSPACE), 932 Parker St suite 2, Berkeley, CA 94710, USA
}

Received 5 April 2021 / Accepted 25 September 2021

\section{ABSTRACT}

\begin{abstract}
Aims. We use multi-spacecraft observations of individual type III radio bursts to calculate the directivity of the radio emission. We compare these data to the results of ray-tracing simulations of the radio-wave propagation and probe the plasma properties of the inner heliosphere.

Methods. We used ray-tracing simulations of radio-wave propagation with anisotropic scattering on density inhomogeneities to study the directivity of radio emissions. Simultaneous observations of type III radio bursts by four widely separated spacecraft were used to calculate the directivity and position of the radio sources. The shape of the directivity pattern deduced for individual events is compared to the directivity pattern resulting from the ray-tracing simulations.

Results. We show that simultaneous observations of type radio III bursts by four different probes provide an opportunity to estimate the radio source positions and the directivity of the radio emission. The shape of the directivity varies from one event to another and it is consistent with anisotropic scattering of the radio waves.
\end{abstract}

Key words. Sun: radio radiation - scattering

\section{Introduction}

Solar type III radio bursts, which are produced by electron beams that are propagating along open magnetic field lines in the corona and interplanetary medium, are among the most intense radio sources in the kilometric range (Wild 1950, 1967). While the precise mechanism of their production has not yet been established, it is widely accepted that type III bursts originate via a plasma emission mechanism in which the fast electrons form a bump-on-tail instability, which then excites Langmuir waves at the local plasma frequency (Ginzburg \& Zhelezniakov 1958; Melrose 1980). These Langmuir waves are then transformed into electromagnetic radiation by mechanisms which are not yet fully understood (Sturrock 1964; Zheleznyakov \& Zaitsev 1970; Smith et al. 1976; Melrose 1987; Dulk 1985; Tkachenko et al. 2021).

Radio waves propagate through the solar corona and interplanetary medium, where they are both refracted and scattered by turbulent plasma processes, with radio-wave scattering on density fluctuations playing a dominant role in the propagation effects (Kontar et al. 2017; Kuznetsov et al. 2020). The observed properties of the radio sources are, therefore, a combination of their intrinsic properties at the emission site and these propagation effects. Radio-wave scattering causes both an increase in the radio source sizes and a very wide emission diagram responsible for the detection of type III radio bursts at all angles in the heliosphere (Steinberg et al. 1984, 1985; Bonnin et al. 2008), a shift of the radio source positions (Fokker 1965; Chrysaphi et al. 2018), and a widening of the intensity time profiles of the radio bursts (Krupar et al. 2018; Kontar et al. 2019).

The effect of radio-wave scattering on solar radio bursts has been studied using ray-tracing simulations (Steinberg et al. 1971; Thejappa \& MacDowall 2008; Krupar et al. 2018, 2020), where isotropic scattering is assumed. However, recent observational results strongly suggest that the properties of solar radio emissions cannot be successfully explained on the 
basis of isotropic scattering (Kontar et al. 2019; Chen et al. 2020). Kontar et al. (2019) presented the results of ray-tracing simulations of radio-wave propagation in the heliosphere, assuming the anisotropic scattering of the radio-waves. These authors showed that scattering was at the origin of the broadening of radio source sizes and radio-burst time profiles, but also that, on average, both properties could only be simultaneously described when anisotropic radio-wave scattering was considered.

The first measurements of the directivity of type III radio bursts were performed by Caroubalos \& Steinberg (1974), Caroubalos et al. (1974), using simultaneous observations from the Earth and from the probe Mars-3, at $169 \mathrm{MHz}$. At lower frequencies (100-500 kHz), Dulk (1985) and Lecacheux et al. (1989) reported that type III radio bursts were detected by instruments irrespective of the position of the radio source. The first stereoscopic directivity measurements were reported by Hoang et al. (1997), who used a combination of ground-based observations from the ARTEMIS spectrograph around $150 \mathrm{MHz}$ and space observations from the radio receiver on board Ulysses (up to $1 \mathrm{MHz}$ ). The first stereoscopic measurements, in the same frequency range, aimed at studying the radio burst directivity were performed by Bonnin et al. (2008), using observations from the Ulysses and Wind spacecraft. Bonnin et al. (2008) used the observations of more than 2000 radio bursts, observed simultaneously by both probes, to statistically derive the directivity of the type III radio bursts between 80 and $1000 \mathrm{kHz}$. These authors confirmed the frequency dependence of the averaged radio burst directivity that was observed by Hoang et al. (1997). These studies had to rely on a statistical analysis of a large sample of bursts, as they had access to only two simultaneous observations of a single event: the directivity profile is therefore calculated using hundreds to thousands of radio flux ratios. With the launch of Solar Orbiter (Müller et al. 2020) and Parker Solar Probe (PSP, Fox et al. 2016), radio measurements at two different points in the heliosphere can be added to the already existing measurements of STEREO-A (Solar Terrestrial Relations Observatory, Kaiser 2005) and Wind (Ogilvie \& Desch 1997), providing, for the first time, an opportunity to study radio emission directivity for single type III radio bursts. In this paper, we present the analysis of five events observed in the early phase of the Solar Orbiter mission (July and November 2020). These observational measurements are compared to the predictions of ray-tracing simulations of the radio-wave propagation with anisotropic radio-wave scattering on turbulent fluctuations of the ambient plasma density. The ray-tracing simulation results are presented in Sect. 2 and the observations are presented in Sect. 3. The comparison between the simulation results and the observed properties of the type III bursts, as well as the implications regarding the radio emission directivity and the properties of the ambient plasma are presented in Sect. 4.

\section{Directivity of radio emission from radio-wave propagation simulations}

\subsection{Simulation setup}

We performed simulations of radio-wave propagation in the heliosphere using the ray-tracing simulations described in Kontar et al. (2019). The ray-tracing simulations detail the propagation of photons through the interplanetary medium, where anisotropic density fluctuations (and, thus, anisotropic scattering) can be assumed. A radio source is placed at a given distance from the solar surface and the corresponding plasma frequency

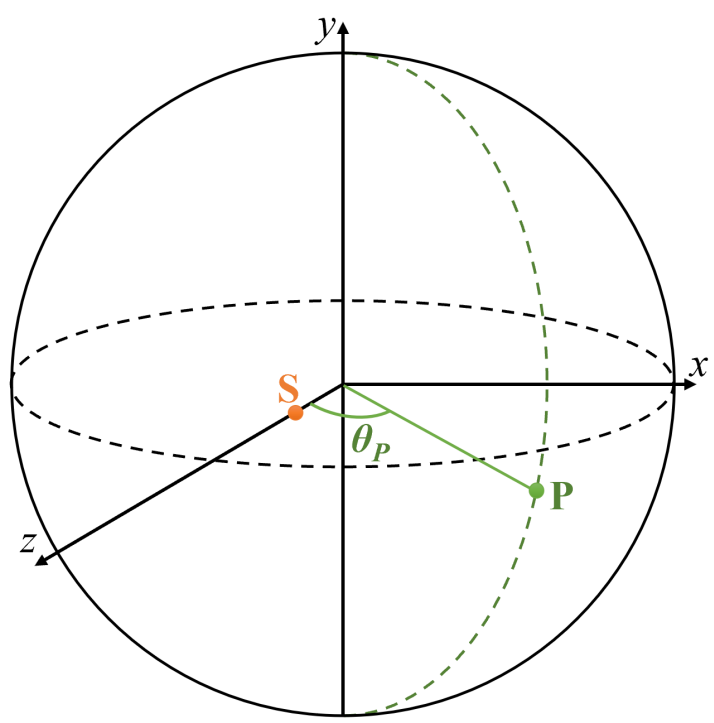

Fig. 1. Geometry of the simulation: radio source $S$ is emitted on the $z$ axis. The angle $\theta_{P}$ is the angle between the direction of the radio source $S$ (the $z$-axis) and the direction of an observer at position $P$. In this paper, the radio source is always situated on the $z$-axis while the probe $P$ has different positions in the $3 \mathrm{D}$ space.

is deduced from the position of the source, assuming a density model. We used the density model described in Kontar et al. (2019):

$n(r)=4.8 \times 10^{9}\left(\frac{R_{s}}{r}\right)^{14}+3 \times 10^{8}\left(\frac{R_{s}}{r}\right)^{6}+1.4 \times 10^{6}\left(\frac{R_{s}}{r}\right)^{2.3}$

where $n(r)$ is the plasma density in $\mathrm{cm}^{-3}, r$ is the position of the source, and $R_{S}$ is the solar radius.

The radio frequency is usually set to a factor of 1.1 or 1.2 (for fundamental emission), or 2 (harmonic emission) of the plasma density, and it remains constant during the radio-wave propagation.

During their propagation, radio-waves are subject to scattering due to turbulent fluctuations of the ambient plasma density. An anisotropic spectrum of density fluctuation $S(\mathbf{q})$, with q being the wave-vector of electron density fluctuations, is assumed, with an axial symmetry. The spectrum is thus parameterised as:

$S(\mathbf{q})=S\left(\sqrt{q_{\perp}^{2}+\alpha^{-2} q_{\|}^{2}}\right)$

where $\alpha$ is the anisotropy factor defined as the ratio of perpendicular to parallel correlation lengths: $\alpha=h_{\perp} / h_{\|}$. When $\alpha<<1$, the spectrum of density fluctuations is dominated by the fluctuations in the perpendicular direction. In the case of isotropic scattering, $\alpha=1$.

The photon propagation is simulated until photons reach a distance from the Sun, where both refraction and scattering become negligible (i.e. when the photon frequency, which is kept constant in the simulations, becomes much larger than the local plasma frequency) or until they reach a distance of 1 au. For each photon, at the end of the simulation, the time, $t$, of arrival and its position, $\mathbf{r}$, and wave-vector, $\mathbf{k}$, are recorded in a Sun-centred coordinate system. 


\subsection{Anisotropic scattering and directivity}

We summarise the geometry we used to analyse the results of the ray-tracing simulation in Fig. 1. The direction of propagation of the radio source, the $z$-axis, is a symmetry axis for the directivity of the radio emission. Therefore, the directivity can be described as a function of the angle $\theta_{P}$ defined in the figure, or of its cosine, $\mu=\cos \left(\theta_{P}\right)$. In our study, as in past studies (e.g. Hoang et al. 1997; Bonnin et al. 2008), we assume that the directivity follows an exponential shape as a function of $\mu$ :

$F(\mu)=C_{0} \exp \left(-\frac{(1-\mu)}{\Delta \mu}\right)$,

where $C_{0}$ is a normalization constant and $\Delta \mu$ is the key parameter controlling the shape of the radio emission directivity pattern. In the simulations, the parameter $\mu$ is calculated as $\mu=k_{z} /|\mathbf{k}|$.

The radio emission directivity pattern depends on the anisotropy factor $\alpha$. We recall here that $\alpha=1$ for isotropic scattering and that $\alpha<1$ results in a stronger scattering in the perpendicular direction. Smaller values of $\alpha$ should therefore lead to a more peaked directivity (Kontar et al. 2019), which translates to smaller values of the parameter $\Delta \mu$ in Eq. (3). We performed ray-tracing simulations of the radio-wave propagation with the following settings: (1) a radio source located at $11 R_{S}$, leading to a plasma density $f_{\mathrm{pe}}=681 \mathrm{kHz}$, and fundamental radio emission emitted at $1.1 \times f_{\mathrm{pe}}{ }^{1}$; and (2) anisotropy factor $\alpha=0.25,0.30,0.40,0.50,0.60,0.75,1.00$.

The directivity obtained at these frequencies for photons arriving around the peak time of the corresponding lightcurve is shown in Fig. 3. To select the photons around the peak time, we generated the lightcurves for the radio emission using the propagation time of each photon resulting from the ray-tracing simulations, and selected photons arriving in the time interval where the lightcurve is above $80 \%$ of the peak time intensity. The directivity obtained with these different simulations, and normalised to the maximum intensity, is displayed in Fig. 2. Each directivity curve was fitted with the model described by Eq. (3), using the mpfitfun procedure to perform the Levenberg-Marquardt least-squares minimisation (Markwardt et al. 2009). The result of this fit is also shown in Fig. 2. As seen in the figure, in the case of isotropic scattering $(\alpha=1)$, the exponential model is a good approximation of the directivity. When we increase the anisotropy of the scattering (by decreasing the ratio $\alpha$ ), the directivity starts to deviate from an exponential shape. However, we keep this model in order to quantify the change of shape of the directivity with the anisotropy factor. As we increase the anisotropy, the directivity distribution gets thinner, as expected, leading to smaller values of the parameter $\Delta \mu$ of the exponential model. In the bottom panel of Fig. 2, the evolution of $\Delta \mu$ resulting from the fits, as a function of the anisotropy factor $\alpha$, is displayed. This evolution is fitted by a model of the form $\Delta \mu \propto e^{\left(a_{0} \alpha\right)}$, which gives $a_{0}=2.2 \pm 0.3$.

\subsection{Evolution of directivity with frequency}

The influence of frequency on the directivity of radio bursts in the radio-wave propagation simulations is examined with the following simulation settings: (1) a radio source located at 5, $8.5,10,13,18$, or $30 R_{S}$, leading to a plasma density of $f_{\mathrm{pe}}=$ $2077,938,765,558,382$, or $211 \mathrm{kHz}$, respectively, and fundamental radio emission emitted at $1.2 \times f_{\mathrm{pe}} ;(2)$ anisotropy factor $\alpha=0.30$.

1 We also simulated a radio sources at a frequency of $1.2 \times f_{\mathrm{pe}}$ and obtained the same directivity profiles.
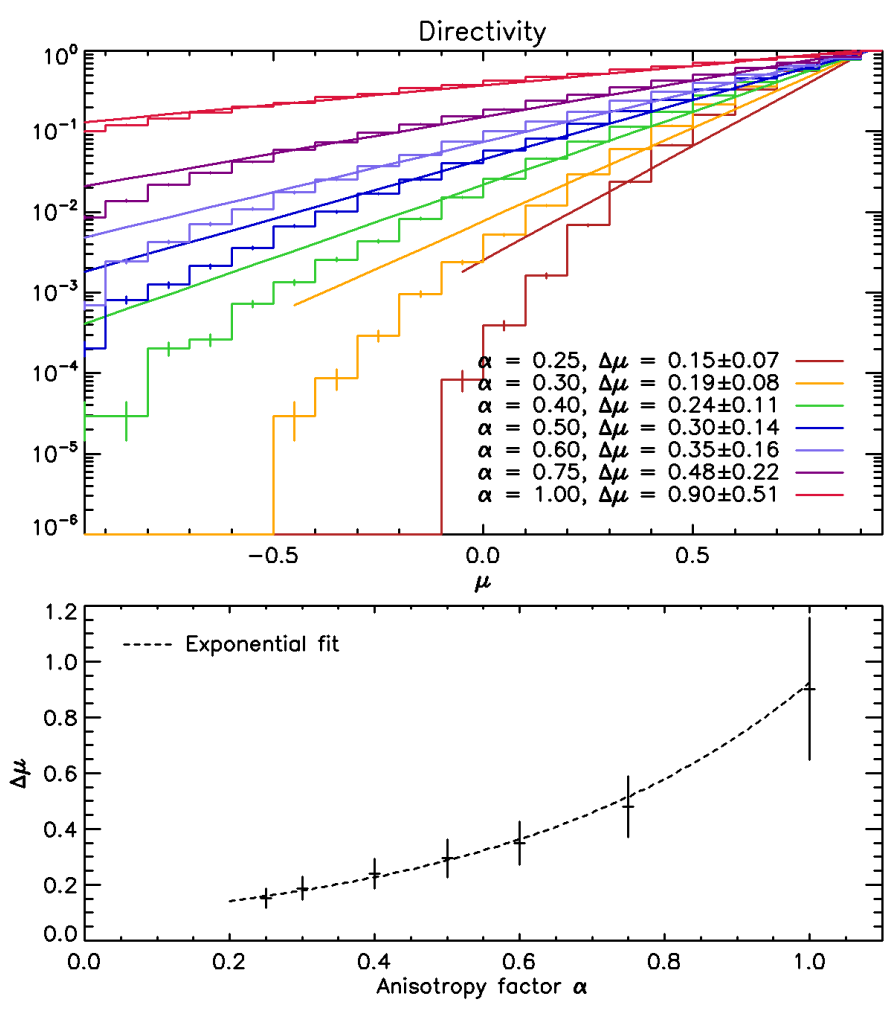

Fig. 2. Effect of the anistropy of density fluctuations on the directivity of the radio emission. Top: normalised directivity as histograms calculated from the results of the simulations (described in Sect. 2.2), with anisotropy factors $\alpha=0.25,0.30,0.40,0.50,0.60,0.75$, and 1.0. The result of the exponential fit is shown as a line for each curve and the values of the parameter $\Delta \mu$ resulting from this fit are displayed in the low right corner. Bottom: evolution of the parameter $\Delta \mu$ with the anisotropy factor $\alpha$. The distribution is fitted with an exponential model: $\Delta \mu \propto e^{\left(a_{0} \alpha\right)}$, which gives $a_{0}=2.2 \pm 0.3$. The result of this fit is shown as a dashed line.

As described in Sect. 2.2, the directivity shown in Fig. 3 is obtained here for photons arriving around the peak time. As can be seen in the figure, there is small dependence of the shape of directivity on frequency, with the parameter $\Delta \mu$ decreasing with increasing frequency. We note that this evolution is a small and remains within the error bars for $\Delta \mu$. We also note that when we select all photons (instead of the photons arriving near the peak time of the light curve), the directivity pattern no longer depends on the frequency.

\subsection{Time profiles at different angles}

Radio-wave scattering is responsible for delays in the propagation time and results in a broadening of the time profiles of type III radio bursts. We used the simulations presented in Sect. 2.2 to quantify the time profile broadening introduced by scattering and the effect of both the radio emission frequency and the anisotropy of the scattering process on the time profile widths (or decay times).

An example of time profiles collected in different directions in space (i.e. at different angles from the radio source direction along the $z$-axis) is shown in Fig. 4. As can be seen in the figure, the time profiles cannot be fully described by an exponential decay: there is a break in the slope taking place roughly when the intensity falls below $10 \%$ of the maximum intensity. This behaviour is introduced by the anisotropy in the scattering 


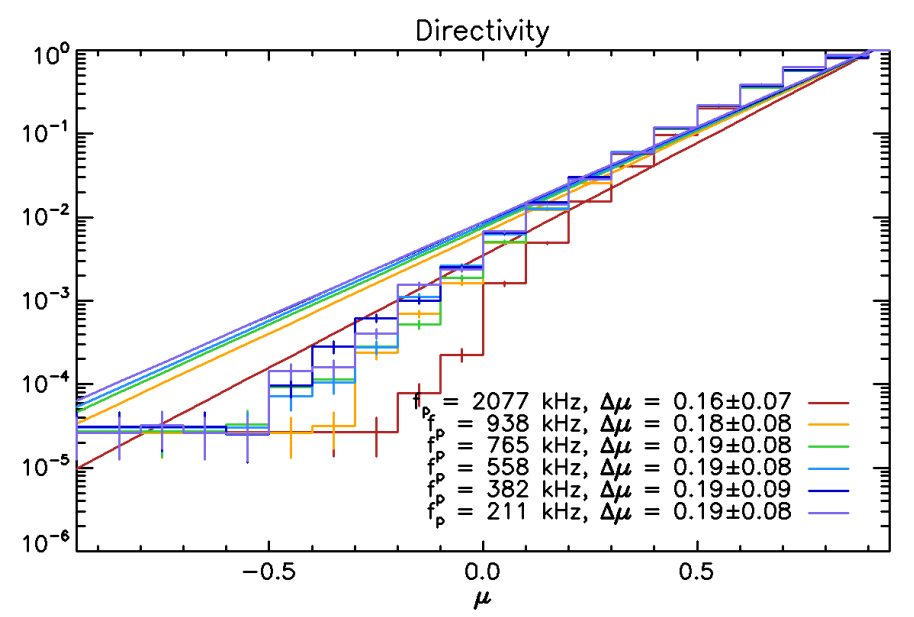

Fig. 3. Normalised directivity as histograms calculated from the results of the simulations described in Sect. 2.3, with anisotropy factor $\alpha=$ 0.30 and plasma frequency of 2077, 938, 765, 558, 382, and $211 \mathrm{kHz}$ in red, orange, green, light blue, dark blue, and purple, respectively. The result of the exponential fit is shown as a line for each curve, and the values of the parameter $\Delta \mu$ resulting from this fit are displayed in the low right corner.

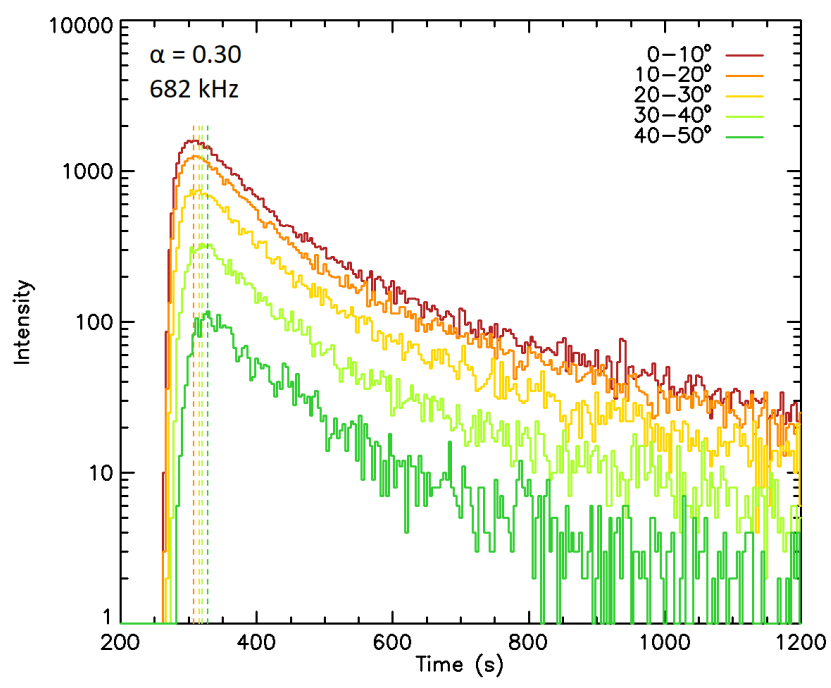

Fig. 4. Example of time profiles obtained for photons collected on a sphere of radius $111 R_{S}$ (where propagation effects become negligible), at different angles $\theta_{P}$, from the simulations of radio-wave propagation from an emission source at $11 R_{S}$ and an anisotropy factor or $\alpha=0.30$. Dashed vertical lines indicate the peak time for each time profile.

process, which is responsible for an 'echo' in the light curve, as described using the same ray-tracing simulations, but at higher frequencies, by Kuznetsov et al. (2020).

For each time profile, the peak time and the full width at half maximum (FWHM) is determined.

Then the decay part of the time profiles is fitted with an exponential model of the form:

$P=P_{0} \exp \left(-\frac{t-t_{0}}{\tau}\right)$

where $\tau$ is the decay time. This fit is performed using the mpfitfun procedure to perform a Levenberg-Marquardt leastsquares minimisation (Markwardt et al. 2009). For this fit, we used only the part of the profile after the peak time, for which the flux is above $10 \%$ of the peak flux.
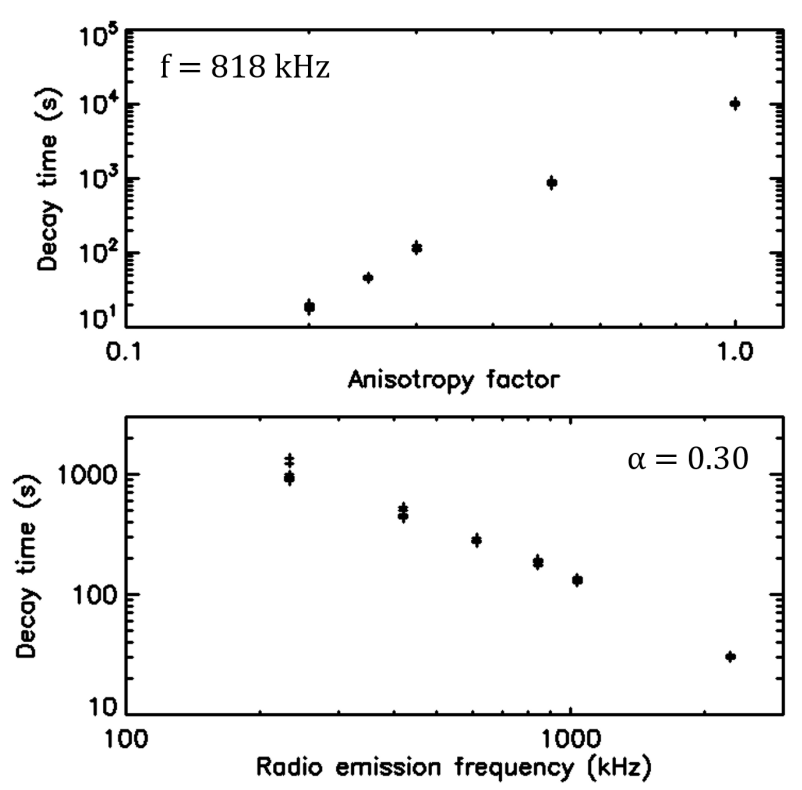

Fig. 5. Evolution of the light curve decay times in the simulations. Top: evolution of the decay time as a function of the anisotropy factor $\alpha$, for emissions at $818 \mathrm{kHz}$. Bottom: evolution of the decay time as a function of the radio emission frequency (assuming fundamental emission) for an anisotropy factor of 0.3 . On each plot, the decay time is determined at different angles from the radio source.

The evolution of the decay time with the anisotropy factor and the radio frequency is shown in Fig. 5. This analysis demonstrates that: (1) the decay time does not vary significantly from one point of view in space to another (from one angle to another); (2) the decay time varies significantly with the anisotropy factor $\alpha$ : as shown in the top panel of Fig. 5, the decay time increases significantly as the anistropy factor increases towards $\alpha=1$, as reported in Kontar et al. (2019); (3) the decay times vary significantly with the radio emission frequency, as shown in the bottom panel of Fig. 5, whereas the decay time decreases as the frequency increases, as also reported in Kontar et al. (2019).

\section{Multi-spacecraft observations of type III radio bursts}

In this section, we describe briefly the space-born radio instruments used in this study and the corresponding data, which have all been calibrated to radio Solar Flux Units (SFU, $1 \mathrm{SFU}=10^{-22} \mathrm{~W} \mathrm{~m}^{-2} \mathrm{~Hz}^{-1}$ ). All these instruments operate in the radio kilometric range, with corresponding frequencies spanning between a few $\mathrm{kHz}$ to $10-20 \mathrm{MHz}$, just below the ionospheric radio cutoff. We then go on to describe our observations and the results of our analyses.

\subsection{Descriptions of the instruments}

We used radio observations embarked on board the Wind (Ogilvie \& Desch 1997), the STEREO-A (Kaiser 2005), the Parker Solar Probe (Fox et al. 2016), and the Solar Orbiter (Müller et al. 2020; Zouganelis et al. 2020) spacecraft.

On Wind, the Waves experiment (Bougeret et al. 1995) carries three electric dipole antennas; two of them are co-planar and orthogonal wire dipole antennas in the spin-plane, whereas the other is a rigid spin-axis dipole. We used the Wind/Waves demodulated data, derived from a so-called direction-finding 

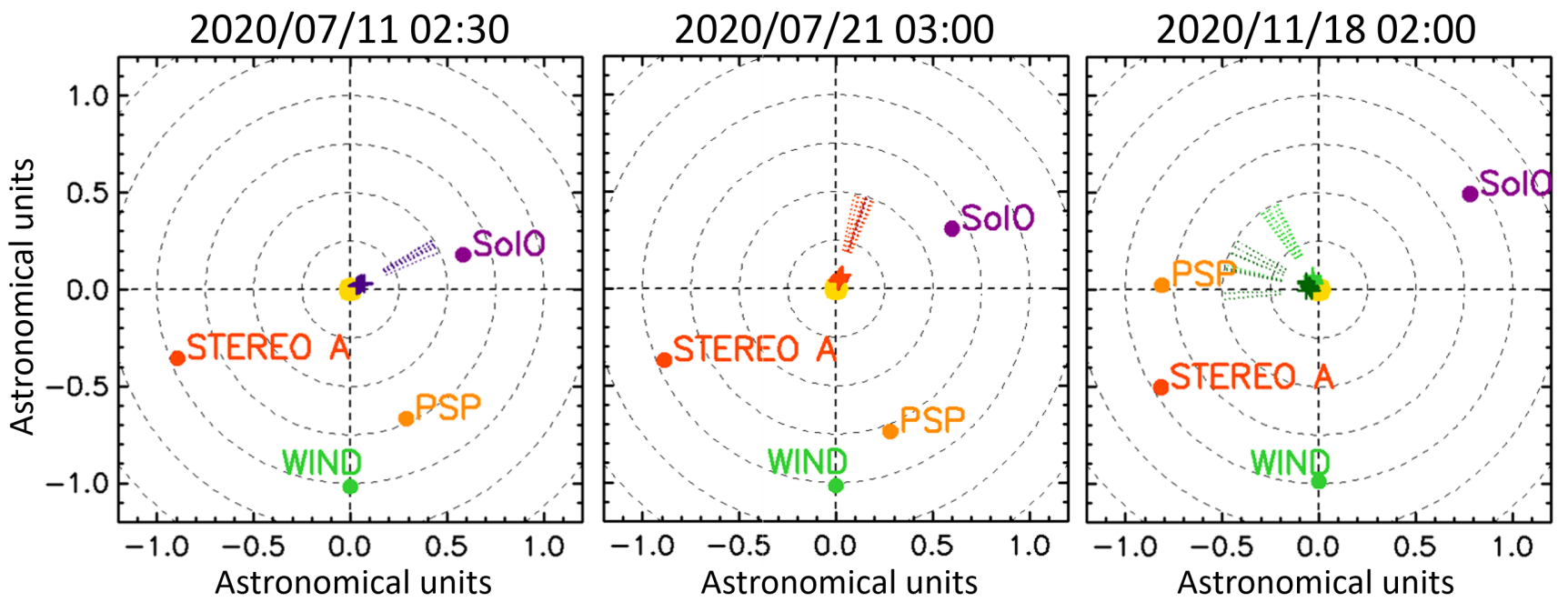

Fig. 6. Position of Solar Orbiter, Parker Solar Probe, STEREO-A and Wind projected in the plane of the Heliocentric Earth Ecliptic (HEE) coordinate system. Left: position on 11 July 2020 at 02:30 UT. The position of the radio source is shown as crosses near the Sun. Middle: position on 21 July 2020 at 03:00 UT. The positions of the radio sources from the two events of that day are indicated by coloured crosses near the Sun. Since both events are from the same region, they are superimposed. Right: position on 18 November 2020 at 02:00 UT. The position of the radio sources for the two events of that day are shown as coloured crosses near the Sun. On this day there is a difference between the location of the radio sources from the first event and from the second event. The determination of the radio source location is described in the text. On each plot, dotted lines are used to point the direction of the radio sources.

method by Manning \& Fainberg (1980) and calibrated using the radio galaxy background (Zaslavsky et al. 2011). This technique provides the absolute radio flux of the observed sources, as well as their polarization properties, directions, and sizes.

On STEREO-A, the S/Waves instrument (Bougeret et al. 2008) performs radio measurements using three orthogonal antenna monopoles. This time, the calibration was based on direction finding techniques specific to three-axis stabilised spacecraft (Cecconi et al. 2008; Krupar et al. 2012, 2016)).

On Parker Solar Probe, we used data recorded by the Radio Frequency Spectrometer (RFS, Pulupa et al. 2017; Bale 2020) connected to the FIELDS electric antennas (Bale et al. 2016). On PSP, the full direction finding radio data pipeline is still in development. However, for sources observed in the frequency range we use in this study (from 400 to $1000 \mathrm{kHz}$ ), the heliospheric locations $\left(12-6 R_{s}\right.$, using the Leblanc et al. 1998 density model) are such that the angles between their $k$-vectors and the radial direction is negligible. Under these conditions, the radio flux can be defined as the sum of the power spectral densities measured by each of the two crossed dipoles, multiplied by their respective $\sin \left(\theta_{\mathrm{Di}}\right)^{2}$, with $\theta_{\mathrm{Di}}$ being the angle between the radial and the given FIELDS dipole. For the events studied here, the values of $\theta_{\mathrm{Di}}$ range between 66 and $81^{\circ}$. The conversion from power spectral densities to radio fluxes in SFU is obtained using the gains as defined by Maksimovic et al. (2020a).

Finally, on Solar Orbiter, we used radio data recorder by the radio receiver part of the RPW (Radio and Plasma Waves) instrument (Maksimovic et al. 2020b). As for PSP, the full directionfinding radio data pipeline is also in development for RPW. Therefore, we made the same assumptions for the directions of the radio sources' $k$-vectors. The only difference is that, contrary to PSP, the RPW antenna dipoles are always perpendicular to the radial direction. We therefore define the radio flux for $\mathrm{SO}$ as being two times the power spectral density as measured by one dipole (the factor of 2 comes from the fact that we assume the source to be non-polarised), multiplied by the calibration gains that have been extensively described by Vecchio et al. (2021).

\subsection{Observations}

For this study, we selected five type III radio bursts that were observed by four widely-separated spacecraft: the first event was observed on 11 July 2020 at about 02:30 UT, the second and third events were observed on 21 July 2020 around 03:00 and 07:00 UT, respectively, and the fourth and fifth events were observed on 18 November 2020 at about 02:00 and 22:30 UT, respectively. The positions of the probes on each day are displayed in Fig. 6. The dynamic spectra of the radio fluxes in SFU for the four spacecraft for the event on 11 July 2020 at about 02:30 UT are given in Fig. 7. As for the other events, we chose to analyse well-defined and isolated type III bursts, which are clearly visible on the four probes.

We selected eight frequency channels for analysing the radio emission, namely: 411, 430, 511, 635, 663, 755, 788, and $979 \mathrm{kHz}$. These particular frequencies were chosen because they correspond to clean RPW frequencies that are not polluted by the strong electromagnetic emission from the Solar Orbiter platform (Maksimovic et al. 2021)

Figure 8 displays temporal variations of the radio fluxes at $634.5 \mathrm{kHz}$ for the four spacecraft and for the event on $11 \mathrm{July}$ 2020 at about 02:30 UT. For each of these light curves, the median of the radio signal on an interval of 30 minutes before 2:20 UT has been removed. All remaining light curves have a classic shape, rapidly reaching a maximum and decaying exponentially. For each of the probes, the time of the peak flux is indicated by the vertical dashed lines.

\subsubsection{Decay times of the type III radio fluxes}

The decay time of the radio bursts was determined at each of the eight selected frequencies by fitting an exponential curve to the decaying part of the burst, using the same model as the one used to analyse the light curves from the simulations (Eq. (4)). The interval chosen to perform the fit is from the peak time to the time when the flux falls below $10 \%$ of the peak intensity. The 


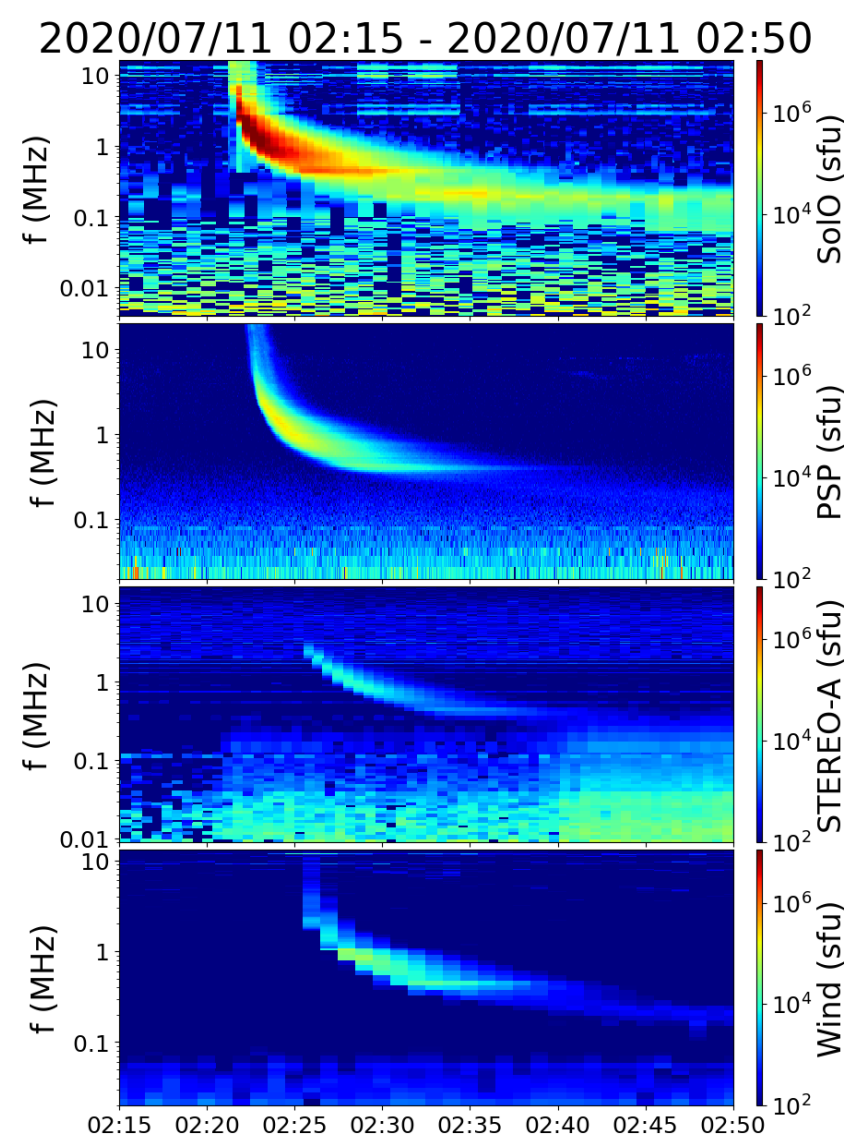

Fig. 7. Dynamic spectrum of the radio fluxes in SFU for the four spacecraft for the event on 11 July 2020 at about 02:30 UT. Top to bottom: Solar Orbiter, Parker Solar Probe, STEREO-A, and Wind.

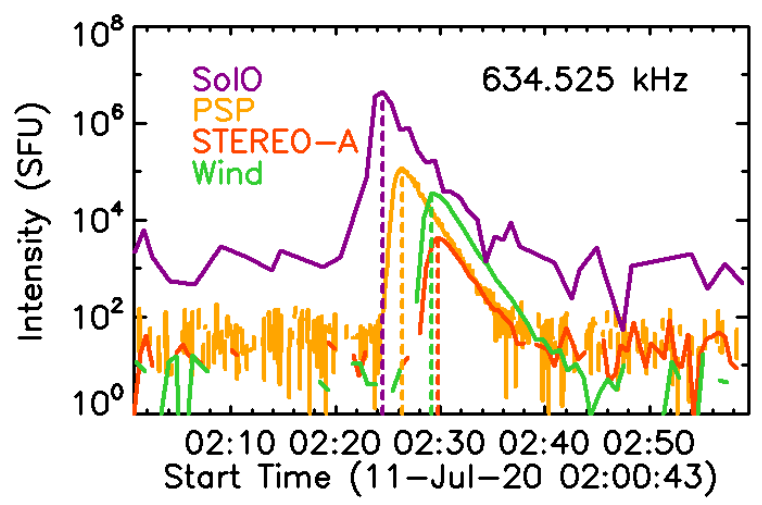

Fig. 8. Light curves of the radio fluxes at $634.5 \mathrm{kHz}$ for the event on July 11 2020, observed at Solar Orbiter, Parker Solar Probe, STEREO$\mathrm{A}$, and Wind. For each of these light curves, the median of the radio signal on an interval of $30 \mathrm{~min}$ before 2:20 UT has been removed. For each of the probes, the time of the peak flux is indicated by the vertical dashed lines.

result of these fits is shown in Fig. 9. As expected, the decay time decreases with increasing frequency. However, there is no clear variation of the decay times between the different events. These observed decay times are aligned with previous measurements of the evolution of the decay time with frequency, such as the compilation of measurement fitted in Kontar et al. (2019), or the recent observations of 30 type III bursts by STEREO and Parker Solar Probe presented in Krupar et al. (2020).

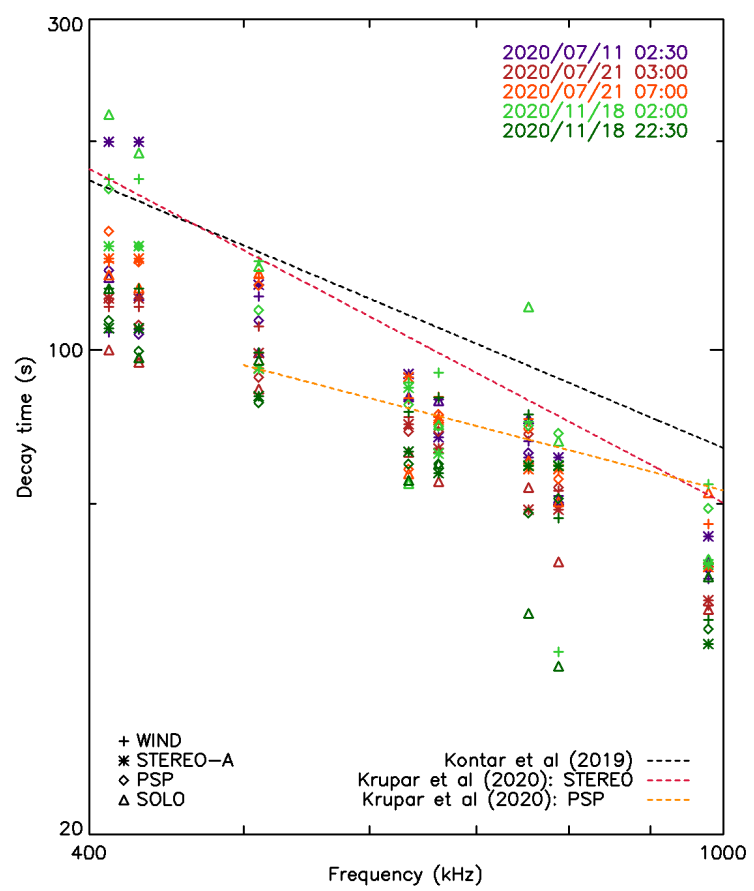

Fig. 9. Decay times of the radio bursts presented as a function of the frequency of the radio emission. Different colours show different type III bursts and different symbols show the different probes. The dashed black line show the result of the fit to the data performed by Kontar et al. (2019) on several data sets. The dashed red and orange lines show the result of the fit on the distribution of decay times from 30 radio bursts observed by STEREO and Parker Solar Probe, respectively (Krupar et al. 2020)

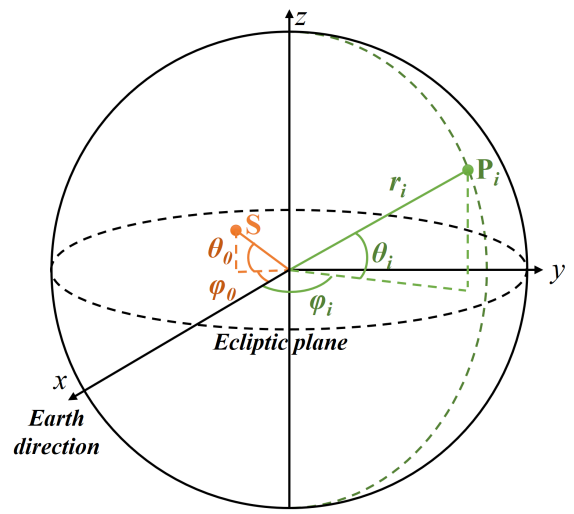

Fig. 10. Position of the radio source and probe $i$ in the HEE coordinate system: longitudes are noted with angle $\varphi$, latitudes with angle $\theta$.

\subsubsection{Directivity fit to observations}

Simultaneous observations of type III radio bursts with four different observation angles allow for a determination of the directivity of the radio emission. While the position of each instrument is well known, the position of the radio source is an unknown parameter. We therefore used the radio flux measurements at the four spacecraft to determine both the shape of the radio emission directivity pattern and the location of the radio source. The geometrical used is illustrated in Fig. 10: the positions of the probes are given in the Heliocentric Earth Ecliptic (HEE) coordinate system, where the $z$-axis is the solar rotation axis and the $x$-axis is in the plane containing the $z$-axis and Earth; the coordinates $\left(r_{i}, \varphi_{i}, \theta_{i}\right)$ correspond to the distance to the Sun, 


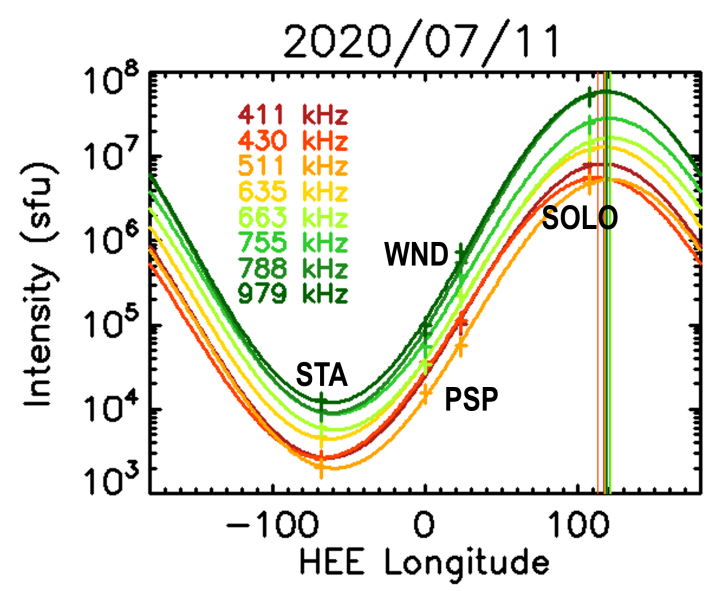

Fig. 11. Directivity fit on the peak fluxes measured during the 11 July 2020 event at different frequencies indicated with different colours. STEREO-A, WIND, Parker Solar Probe, and Solar Orbiter measurements are indicated by crosses and labelled as STA, WND, PSP, and SOLO respectively. The vertical lines show the position of the radio source in longitude as determined from the directivity fit.

as well as the longitude and latitude of probe $i$ in this coordinate system.

During our observations, the different spacecraft are at different distances from the radio source. Since the radio source remains very close to the Sun, we assume that we can correct the flux intensity of each spacecraft for their relative distance to the radio source by using the heliocentric distance of the spacecraft. The fluxes corrected for the different distances are noted as $I_{\mathrm{au}}$ since they represent the radio flux that would have been measured if the spacecraft was positioned at 1 au from the Sun.

The radio flux at 1 au depends on the position of the observation point with respect to the radio source, the intensity of the radio emission, and the directivity of the emission. Therefore, it can be described as a function of the source position $\left(\varphi_{0}, \theta_{0}\right)$, the probe position $(\varphi, \theta)$, and the parameter $\Delta \mu$ for the directivity. The directivity is modelled using Eq. (3) with $\mu=\cos \left(\varphi-\varphi_{0}\right) \cos \left(\theta-\theta_{0}\right)$ and $C_{0}$ the maximum intensity, when $\varphi=\varphi_{0}$ and $\theta=\theta_{0}$.

In the case of multi-spacecraft observations of type III radio bursts, the position of the probes $\left(\varphi_{i}, \theta_{i}\right)$ is known for each probe $i$, while the position of the source $\left(\varphi_{0}, \theta_{0}\right)$ and the parameters $\Delta \mu$ and $C_{0}$ are the four unknowns. Using the MPFIT procedure, we fit a directivity profile to best represent the radio fluxes measured at the different spacecraft. The result of the fit gives both a measurement of the directivity parameter $\Delta \mu$ and a location of the radio source $\left(\varphi_{0}, \theta_{0}\right)$, independent of other methods to locate the source (e.g. triangulation).

For the events presented in this paper, the probes are all located close to the ecliptic plane, at low latitudes; therefore, there are poor constraints on the latitude of the radio source $\theta_{0}$ and this parameter was thus kept fixed to 0 for the directivity fit. The directivity model is fitted using the peak intensity at each of the eight frequencies selected, resulting in eight directivity profiles for each event. An example of the directivity fit shown in the ecliptic plane is displayed in Fig. 11. The main results of these fits are the amplitude of the radio emission directivity pattern of the radio burst, characterised by the parameter $\Delta \mu$, and the position of the radio source (longitude, with the assumption of a source in the ecliptic plane).

Changes in the level of uncertainties in the measured fluxes at the different probes do not significantly affect the results of the

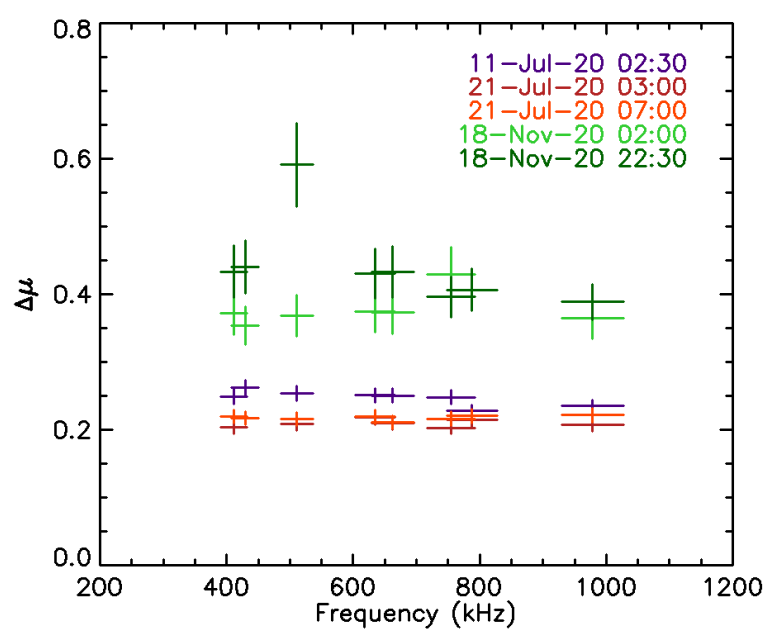

Fig. 12. Parameter $\Delta \mu$ determined during the directivity fitting of the peak fluxes for the five radio bursts selected.

directivity fit: while the uncertainty on the parameters is linked to the uncertainty on the measured fluxes, the parameter values themselves do not change significantly. Moreover, even if the fluxes from the RPW and FIELDS are not determined with the full direction-finding radio data pipelines in this paper, no significant change to the results presented here should be expected once the final calibration to the data is applied.

In order to determine the radio source location, we use the results of our directivity fit, which provide the longitude and latitude of the source, and estimate the radial distance of the source to the Sun using a density model. In this paper, the latitude of the source is assumed to be 0 , and we used the density model described by Eq. (1), assuming that the radio emission is emitted at the fundamental frequency. The resulting source positions are displayed in each panel of Fig. 6 as coloured crosses for each event. On 21 July 2020, the radio sources positions from the two type III radio bursts overlap. On 18 November 2020, the radio sources positions from the two type III radio bursts are slightly shifted, and it is likely that these sources are emitted by electron beams originating from the same active region. For all events, the deduced locations of the radio emission sources roughly correspond to a longitude at which active regions are present in the synoptic maps of the magnetic field of the solar surface.

The amplitude of the radio burst directivity, determined from the directivity fit, is displayed as a function of frequency in Fig. 12. As seen in the figure, the values of the parameter $\Delta \mu$ can vary significantly from one event to another. However, no clear variation with frequency is found for the parameter $\Delta \mu$. The mean values of the parameters and the standard deviation for each event are displayed in Fig. 1. For the first three events, the value of $\Delta \mu$ is found to be between 0.21 and 0.25 , with very little deviation. For the last two events, the value of $\Delta \mu$ is found to be around 0.4 , but with greater uncertainties.

\section{Discussion}

\subsection{Comparison of observational and simulation results}

Ray-tracing simulations were used to explore the variations of radio burst properties with respect to the variation of several parameters of the ambient plasma. As shown in Kontar et al. (2019) (and recalled in this paper) the time profile and directivity of the radio emission depends on the anisotropy factor 
Table 1. $\Delta \mu$ averaged over the different frequencies for each event, and corresponding standard deviation (The standard deviation here is calculated over the different frequencies for each individual event.).

\begin{tabular}{lcc}
\hline \hline Event & Mean value of $\Delta \mu$ & Standard deviation \\
\hline 2020/07/11 02:30 & 0.25 & 0.01 \\
2020/07/21 03:00 & 0.21 & 0.01 \\
2020/07/21 07:00 & 0.22 & 0.01 \\
2020/11/18 02:00 & 0.4 & 0.2 \\
2020/11/18 22:30 & 0.4 & 0.4 \\
\hline
\end{tabular}

introduced to account for anisotropic scattering of radio-waves on density fluctuations of the ambient plasma. Using the results of ray-tracing simulations with different anisotropy factor $\alpha$, we established a clear relation between the shape of the radio emission directivity, characterised by the parameter $\Delta \mu$ and the level of anisotropy. We also show here that simulation results suggest that this parameter $\Delta \mu$ exhibits only a weak dependence on the radio emission frequency.

The directivity of type III radio bursts can only be determined from observations when simultaneous observations of type III radio bursts are available from several different vantage points. In this paper, we use observations at four different probes, which are all located close to the ecliptic plane: we therefore limited our analysis in this plane and made the assumption that the radio sources were emitted in the ecliptic plane. For the first three events, the averaged values of $\Delta \mu$ lie between 0.21 and 0.25 , which corresponds to anisotropy factors $\alpha$ between 0.36 and 0.44 in the simulations presented in Sect. 2. For the last two events, the average value of $\Delta \mu$ is of 0.4 , corresponding to an anisotropy factor of 0.6.

As shown in Fig. 5, with an anisotropy factor as low as $\alpha=0.30$, the decay times from the simulations are higher than the observed decay time by roughly a factor of 2 . Given the uncertainties in the measurements and the approximations made in the simulations, this retains a good agreement. However, given that higher anisotropy factors lead to increased decay times, it may be more difficult to explain the observed decay times for the last two events for which the anisotropy factor is believed to be around 0.6. Thus, a further investigation of the influence of the assumptions and other parameters in the simulation are necessary to completely explain these observations. We also noted that anisotropic scattering of radio waves introduced an 'echo' that is manifested in the light curves as a deviation from the exponential shape of the decay part of the curve. However, this deviation is subtle and only visible after the time when the intensity drops roughly below $10 \%$ of the peak intensity. This property might therefore be challenging to discern in the observations.

\subsection{Individual radio burst properties compared to statistical results}

This paper reports on multi-spacecraft observations of single type III radio bursts, whose properties can be compared to the results of previous studies that have examined such parameters statistically.

As discussed in Sect. 3, the decay times of the radio bursts show similar (or slightly lower) values as the decays times reported for STEREO and Parker Solar Probe observations (Krupar et al. 2020) or as compiled in Kontar et al. (2019) based on a range of studies. The decreasing trend of the decay time with increasing frequency is also observed on a single event

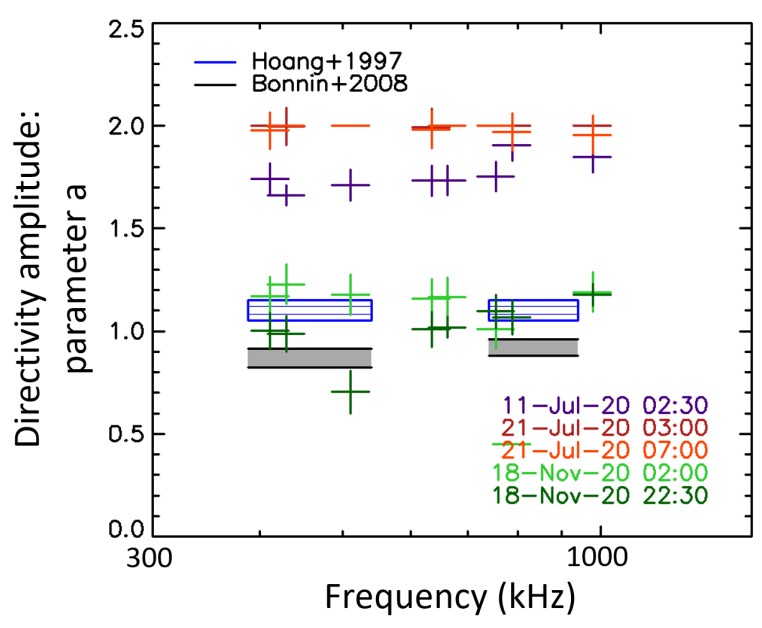

Fig. 13. Parameter $a$ describing the amplitude of the directivity, for the model described by Eq. (5), and determined by a fit of this model to the peak fluxes for the five radio bursts selected, shown as coloured crosses. Previous results obtained in statistical studies of flux ratios of type III radio bursts by Bonnin et al. (2008) and Hoang et al. (1997) are also shown as squared boxes.

basis. We show here that this decay times does not vary significantly with the angle between the source and the point of observation (probe position).

On the other hand, the directivity profiles determined in this study for individual radio bursts can be compared to the directivity of radio bursts determined statistically on thousands of events, as reported by Bonnin et al. (2008), Hoang et al. (1997). In their study, the average directivity of radio burst was determined by fitting the distribution of flux ratios observed by two probes, for thousands of events. A slightly different model was then used for the directivity, taking the form:

$D\left(\varphi^{\prime}\right)=C_{0} 10^{\left(a\left(\cos \varphi^{\prime}-1\right)\right)}$

where $\varphi^{\prime}$ is the angle between the source direction and the probe position in a source-centered coordinate system. In our analysis, we consider the angles in a sun-centered system. However, since the frequency of our measurements is above $400 \mathrm{kHz}$, the radio sources remain very close to the Sun, and we consider that $\varphi^{\prime}=$ $\varphi$, with $\varphi$ being the difference between the probe longitude and the source longitude. In that specific case, the relation between parameters $a$ and $\Delta \mu$ is simple: $a=(\ln (10) \Delta \mu)^{-1}$.

We used this model described by Eq. (5) to fit the data, following the same method used to calculate the $\Delta \mu$ parameter, but this time to calculate the values of the parameter $a$ that is consistent with our observations, which was then compared to the values of this parameter found by the previous studies. The results are shown in Fig. 13. The values of the parameter are of the same order of magnitude as the ones calculated in the statistical studies, which could be considered to be average values, as it is expected that the values from a single event to another can vary significantly and differ from the average. We also note that the values of the parameter $a$ found by fitting the data verify the expected relation: $a=(\ln (10) \Delta \mu)^{-1}$.

In the frequency range studied here, there is no obvious evolution of the directivity shape with frequency. In particular, Bonnin et al. (2008) and Hoang et al. (1997) reported a variation of the directivity with frequency, which was significant at frequencies below $400 \mathrm{kHz}$. It can be noted that such a dependence is not observed in the ray-tracing simulations; however, at frequencies below $400 \mathrm{kHz}$, it is probable that we can no longer 
assume that the angles that are defined with respect to the centre of the Sun (as it is the case in the simulations) and those defined with respect to the source (as it is in these studies) can be considered to be similar. This change in geometry could therefore account for the frequency dependence of the directivity profiles found in these past results.

We note that the directivity profiles found for the last two events presented here are similar to the results found in the previous studies of Bonnin et al. (2008) and Hoang et al. (1997), while the directivity profile for the first three events is narrower (with higher values of the parameter $a$ ). These observations demonstrate how the directivity profile can deviate from the average values determined by past studies. It is interesting to note that it is possible that the shape of directivity (i.e. the values of $\Delta \mu$ or $a$ ) seems to remain consistent over time: the two events most closely related in time (on 21 July 2020) show almost identical directivity profiles. Since those events also share the same location, this can be interpreted as events for which the solar wind conditions remains the same. These results therefore demonstrate a potential for this type of analysis to probe the plasma and solar wind conditions at the sources of the radio emission in the inner heliosphere.

\section{Conclusion}

The launch of the Solar Orbiter and Parker Solar Probe missions, in combination with the STEREO and Wind missions, have opened the opportunity for the simultaneous observations by four widely-separated spacecraft of solar events, such as type III radio bursts. Multi-spacecraft observations of these radio emissions are used here to study the directivity of single type III radio burst. In the past, directivity measurements could only be performed by a statistical analysis of radio flux ratios from many different bursts, whereas, in the present study, we also provide an estimation of the source locations. As the data presented here were taken early in the Solar Orbiter mission, all measurements are performed close to the ecliptic plane and, therefore, no information on the latitude of the radio sources can be reliably inferred; however, as the Solar Orbiter mission continues, the satellite orbit will increase in inclination in regards to the ecliptic plane (Müller et al. 2020; Zouganelis et al. 2020), providing an opportunity to further study the directivity of the radio emissions in the 3D space for individual events.

In this study, we looked at five different radio bursts and we show that while their directivity could be consistent with the previous averaged results, it could also vary significantly from these averaged values and from one event to another. The two closest events in terms of time and space, namely, on 21 July 2020, show very similar directivity profiles, suggesting that the radio emission was emitted in similar solar wind conditions. This suggests, as we might expect, that the radio emission directivity is significantly affected by the properties of the ambient plasma and solar wind.

Ray-tracing simulations of radio-wave propagation with anisotropic scattering on density fluctuations suggest that the directivity pattern of radio emission strongly depends on the ambient plasma conditions and, in particular, on the anisotropy of the density fluctuations of the plasma. Multi-spacecraft radio emission diagnostics therefore enable us to characterise the anisotropy in the plasma density fluctuations. In the 400$1000 \mathrm{kHz}$ range, we typically probe plasma in the range 5-20 solar radii from the Sun. At this distance range, it will be possible to combine the type of observations presented in this paper with in situ measurements by Parker Solar Probe at its closest approach, and also to get beyond the range explored in situ by the probe.

Acknowledgements. Solar Orbiter is a space mission of international collaboration between ESA and NASA, operated by ESA. Parker Solar Probe was designed, built, and is now operated by the Johns Hopkins Applied Physics Laboratory as part of NASA's Living with a Star (LWS) program (contract NNN06AA01C). Support from the LWS management and technical team has played a critical role in the success of the Parker Solar Probe mission. The Wind/WAVES investigation is a collaboration of the Observatoire de Paris, NASA/GSFC, and the University of Minnesota. S. Musset is supported by an ESA Research Fellowship. V. Krupar acknowledges the support by NASA under grants 18-2HSW0218_2-0010 and 19-HSR-19_2-0143. N. Chrysaphi thanks CNES for its financial support. Data analysis was performed using IDL (Exelis Visual Information Solutions, Boulder, Colorado).

\section{References}

Bale, S. D. 2020, Parker Solar Probe, FIELDS Radio Frequency Spectrometer, RFS, High Frequency Receiver, HFR, Spectra, Level 2 (L2), 7 s and 56 s Data (NASA Space Physics Data Facility)

Bale, S. D., Goetz, K., Harvey, P. R., et al. 2016, Space Sci. Rev., 204, 49 Bonnin, X., Hoang, S., \& Maksimovic, M. 2008, A\&A, 489, 419

Bougeret, J. L., Kaiser, M. L., Kellogg, P. J., et al. 1995, Space Sci. Rev., 71, 231 Bougeret, J. L., Goetz, K., Kaiser, M. L., et al. 2008, Space Sci. Rev., 136, 487 Caroubalos, C., \& Steinberg, J. L. 1974, A\&A, 32, 245

Caroubalos, C., Poquerusse, M., \& Steinberg, J. L. 1974, A\&A, 32, 255

Cecconi, B., Bonnin, X., Hoang, S., et al. 2008, Space Sci. Rev., 136, 549

Chen, X., Kontar, E. P., Chrysaphi, N., et al. 2020, ApJ, 905, 43

Chrysaphi, N., Kontar, E. P., Holman, G. D., \& Temmer, M. 2018, ApJ, 868, 79 Dulk, G. A. 1985, ARA\&A, 23, 169

Fokker, A. D. 1965, Bull. Astron. Inst. Neth., 18, 111

Fox, N. J., Velli, M. C., Bale, S. D., et al. 2016, Space Sci. Rev., 204, 7 Ginzburg, V. L., \& Zhelezniakov, V. V. 1958, Sov. Astron., 2, 653

Hoang, S., Poquerusse, M., \& Bougeret, J. L. 1997, Sol. Phys., 172, 307 Kaiser, M. L. 2005, Adv. Space Res., 36, 1483

Kontar, E. P., Yu, S., Kuznetsov, A. A., et al. 2017, Nat. Commun., 8, 1515 Kontar, E. P., Chen, X., Chrysaphi, N., et al. 2019, ApJ, 884, 122 Krupar, V., Santolik, O., Cecconi, B., et al. 2012, J. Geophys. Res. (Space Phys.), 117, A06101

Krupar, V., Eastwood, J. P., Kruparova, O., et al. 2016, ApJ, 823, L5 Krupar, V., Maksimovic, M., Kontar, E. P., et al. 2018, ApJ, 857, 82 Krupar, V., Szabo, A., Maksimovic, M., et al. 2020, ApJS, 246, 57 Kuznetsov, A. A., Chrysaphi, N., Kontar, E. P., \& Motorina, G. 2020, ApJ, 898, 94

Leblanc, Y., Dulk, G. A., \& Bougeret, J.-L. 1998, Sol. Phys., 183, 165

Lecacheux, A., Steinberg, J. L., Hoang, S., \& Dulk, G. A. 1989, A\&A, 217, 237

Maksimovic, M., Bale, S. D., Berčič, L., et al. 2020a, ApJS, 246, 62

Maksimovic, M., Bale, S. D., Chust, T., et al. 2020b, A\&A, 642, A12

Maksimovic, M., Souček, J., Chust, T., et al. 2021, A\&A, 656, A41 (SO Cruise Phase SI)

Manning, R., \& Fainberg, J. 1980, Space Sci. Instrum., 5, 161

Markwardt, C. B. 2009, in Astronomical Data Analysis Software and Systems XVIII, eds. D. A. Bohlender, D. Durand, \& P. Dowler, ASP Conf. Ser., 411, 251

Melrose, D. B. 1980, Space Sci. Rev., 26, 3

Melrose, D. B. 1987, Sol. Phys., 111, 89

Müller, D., St. Cyr, O.C., Zouganelis, I., et al. 2020, A\&A, 642, A1

Ogilvie, K. W., \& Desch, M. D. 1997, Adv. Space Res., 20, 559

Pulupa, M., Bale, S. D., Bonnell, J. W., et al. 2017, J. Geophys. Res. (Space Phys.), 122, 2836

Smith, R. A., Goldstein, M. L., \& Papadopoulos, K. 1976, Sol. Phys., 46, 515

Steinberg, J. L., Aubier-Giraud, M., Leblanc, Y., \& Boischot, A. 1971, A\&A, 10, 362

Steinberg, J. L., Hoang, S., Lecacheux, A., Aubier, M. G., \& Dulk, G. A. 1984, A\&A, 140, 39

Steinberg, J. L., Hoang, S., \& Dulk, G. A. 1985, A\&A, 150, 205

Sturrock, P. A. 1964, Type III Solar Radio Bursts, 50, 357

Thejappa, G., \& MacDowall, R. J. 2008, ApJ, 676, 1338

Tkachenko, A., Krasnoselskikh, V., \& Voshchepynets, A. 2021, ApJ, 908, 126

Vecchio, A., Maksimovic, M., Krupar, V., et al. 2021, A\&A, 656, A33 (SO Cruise Phase SI)

Wild, J. P. 1950, Aust. J. Sci. Res. Phys. Sci., 3, 541

Wild, J. P. 1967, Proc. Astron. Soc. Aust., 1, 38

Zaslavsky, A., Meyer-Vernet, N., Hoang, S., Maksimovic, M., \& Bale, S. D.

2011, Radio Sci., 46, RS2008

Zheleznyakov, V. V., \& Zaitsev, V. V. 1970, Sov. Astron., 14, 47

Zouganelis, I., De Groof, A., Walsh, A. P., et al. 2020, A\&A, 642, A3 